\title{
A Hybrid Cache and Prefetch Mechanism for Scientific Literature Search Engines
}

\author{
Huajing Li ${ }^{1}$, Wang-Chien Lee ${ }^{1}$, Anand Sivasubramaniam ${ }^{1}$, and C. Lee Giles ${ }^{1,2}$ \\ ${ }^{1}$ Department of Computer Science and Engineering \\ ${ }^{2}$ The School of Information Sciences and Technology \\ Pennsylvania State University \\ State College, PA 16802, USA \\ \{huali, wlee, anand\}@cse.psu.edu, giles@ist.psu.edu
}

\begin{abstract}
CiteSeer, a scientific literature search engine that focuses on documents in the computer science and information science domains, suffers from scalability issue on the number of requests and the size of indexed documents, which increased dramatically over the years. CiteSeer ${ }^{\mathcal{X}}$ is an effort to re-architect the search engine. In this paper, we present our initial design of a framework for caching query results, indices, and documents. This design is based on analysis of logged workload in CiteSeer. Our experiments based on mock client requests that simulate actual user behaviors confirm that our approach works well in enhancing system performances.
\end{abstract}

\section{Introduction}

Due to the rapid growth of the Web in recent years, Internet has changed our everyday life style dramatically. Various types of web applications have come into shape and begun to service the public, among which maybe the most successful story is the popularity of search engines (i.e. Googl@1 AltaVist施). As one important branch of search engine applications, scientific literature search engines are welcomed because of their dedication in a specific domain, which in result improve the quality of services. Unlike generic search engines such as Google, scientific literature search engines limit their scope in a set of tightly-correlated topics. CiteSeer [2]6|7] is a web-based scientific literature search engine which focuses on computer and information science. On average, CiteSeer receives over 800,000 requests daily, is accessed by over 100,000 unique users monthly, and serves approximately 30 gigabytes of data daily. As the document corpus grows larger and the workload increases, it is observed that CiteSeer suffers from its original design deficiencies, causing long service latencies and larger maintenance costs.

A perceivable performance metric for end-users of search engines is the query response time. Namely, the time it takes for a user to wait before an issued query to be answered and returned. Inside a search engine, queries are sent to the server, which accesses the indices (in the form of inverted lists) to obtain the result set. In addition to the query performance, CiteSeer has another concern as well: the document retrieval

\footnotetext{
${ }^{1}$ http://www.google.com

${ }^{2} \mathrm{http}: / /$ www.altavista.com
}

L. Baresi, P. Fraternali, and G.-J. Houben (Eds.): ICWE 2007, LNCS 4607, pp. 121-136, 2007.

(C) Springer-Verlag Berlin Heidelberg 2007 
efficiency, which represents the time it takes for the system to generate the detail page of a document and present it to the user. As we click on a specific item in a search result, CiteSeer will provide a summary page describing the document, including title, author, abstract, related documents, bibtex, etc. The required data are located in distributed sources, some of which have to be generated by internal algorithms on-the-fly. Considering the huge volume of requests for documents, it is critical to improve the document retrieval efficiency.

Caching techniques have been used to address performance issue of information systems, including search engines. Two levels of caches exist for current search engine applications. Result cache [913] stores previous search results, while index cache [4], on the other hand, stores inverted lists for query terms that have been used. In addition, as an alternative to existing cache replacement policies, prefetching can improve the system performance by fetching result list beforehand.

To better understand the characteristics of CiteSeer, usage logs are analyzed to gain insights into typical user behaviors. In our analysis, we found high shareness and locality in user requests, with popular requests repeating frequently. Existing works mainly apply a cache management policy to the system. With detailed analysis into the request distribution characteristics, we believe such one-policy scheme lacks the flexibility to reflect the distribution features. Hence, we propose a hybrid cache management scheme, whose performance can be optimized by tuning parameters according to the summarized request distributions. In addition, our workload analysis reveals that high correlation exists for requests to CiteSeer, which motivates us to incorporate a correlationbased prefetching mechanism into the framework. This unique function is not available in existing caching mechanisms for search engines. Also, we include document caching in improving document retrieval latency.

Previously proposed caching approaches do not fully exploit the potential of the unique running characteristics exhibited by CiteSeer logs to optimize the system performance. In this paper, we propose and analyze a hybrid cache and prefetch framework for CiteSeer ${ }^{\mathcal{X}}$. Our contributions can be summarized as follows:

- We propose an integrated hybrid caching framework, which is based on our analysis to the the logged CiteSeer trace. The framework is comprised of multiple components, each of which utilizes a combined cache management policy and is dedicated to improve the latencies for a specific group of requests.

- We include the term and document correlation into consideration in predicting future user requests and thus provide prefetch facility before a user actually requests documents.

- We experimentally evaluate the proposed hybrid cache to test its effect based on CiteSeer's actual data and workload. Also, we demonstrate the tuning of cache's running parameters to achieve optimized performance.

The rest of the paper is organized as follows. Our analysis to CiteSeer's usage logs is presented in Section 2. We propose our hybrid caching framework for CiteSeer ${ }^{\mathcal{X}}$ in Section 3 . Section 4 gives our evaluation results and discussions. In Section 5 , we briefly review and compare proposed caching mechanisms in the literature. Finally, Section 6 provides the concluding remarks. 


\section{Workload Analysis}

In order to understand the working nature of CiteSeer, usage logs are parsed and analyzed so that we can have insights into typical user behaviors and evaluate the effectiveness of a system cache. This section summarizes our workload analysis results. Basically, Section 2.1 introduces the preliminary tasks we performed to the usage logs. Section 2.2 gives a statistical summary to basic attributes of the logs. Afterwards, Section 2.3 and Section 2.4 respectively give the request frequency distribution analysis and locality analysis. Next, we studied the correlation between user requests, which is presented in Section 2.5. Finally, we give the analysis summary.

\subsection{Data Preparation}

A typical CiteSeer logging entry consists of five parts: time stamp, request type, parameter (query terms, requested document identifiers, etc), IP address, and agent type. An example is given below:

\subsection{7 event context 017827470446930 ip: 128.255.54. * * Generic}

Although the log format contains all the information needed for the research, some data preparation tasks are necessary. First, irrelevant request types of the study are filtered out. Only three request types are kept, which are (1) document query, (2) document detail, and (3) document download. The latter two request types are treated as the same type in the analysis because they are both requests for document records. The second task we performed is to sessionize the logged trace. In CiteSeer, the system does not record user identities as well as session information. We use the following heuristics to differentiate sessions: (1) The IP address is used as the user identity. (2) A time threshold is used to measure the request interval from a same IP address. In our experiment, we set the time threshold to be 1,800 seconds (30 minutes). Finally, it is found the robot requests form a considerable portion of the network traffic. To better understand a user's behavior, robot requests are removed. Basically, we used a robot agent string lis 4 to identify obvious robots. To those robots that do not declare themselves, statistical analysis is performed to identify them. If we find that either the average session length is extremely long (more than 500) or a portion (10\%) of intra-session intervals are particularly small (less than 0.5 second), the IP address is tagged with "robots" because they exhibit odd behaviors from normal human users.

\subsection{Statistical Summary}

Two-week-length CiteSeer logs are collected and analyzed in this paper. Table 1 gives the statistical summary of the usage logs. From the number of requests and the number of the unique requests we can infer that many requests do not repeat frequently (also shown in Section 2.3. Also, we can see that the for most sessions, the session length is limited in a relatively small value. The detailed session length distribution can be found in Figure 1(a), from which we can observe that most sessions have a length of 2 or 3.

\footnotetext{
${ }^{3}$ We suppressed the last section of the IP address.

${ }^{4}$ http://www.pgts.com.au/pgtsj/pgtsj0208d.html
} 
Table 1. Statistical summary of the analyzed logs

\begin{tabular}{|l|l|}
\hline Summary & Trace \\
\hline Number of Document Query Requests & 601,337 \\
\hline Number of Document Retrieval Requests & $2,273,233$ \\
\hline Number of Distinct Document Query Requests & 334,731 \\
\hline Number of Distinct Document Retrieval Requests & 393,416 \\
\hline Average Session Length & 2.839 \\
\hline
\end{tabular}

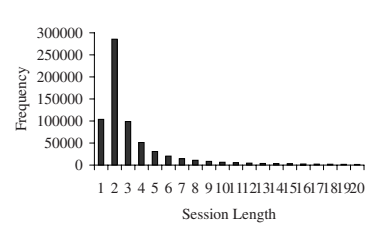

(a) Session length distribution

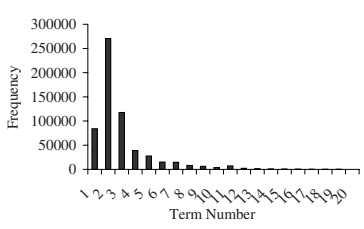

(b) Term number distribution for document queries

Fig. 1. Statistical analysis

We also performed an analysis on the distribution of term numbers in document queries. From Figure 1(b), it can be observed that most queries only contain a small number of terms (less than 10). This finding implies that there is a high possibility that some unique terms are frequently queried.

\subsection{Frequency Distribution}

As we look through the logs, we find that there exist a set of hot query terms and documents in the system. The requests to these terms and documents are very frequent. Correspondingly, the large portion of the requested term corpus and document corpus only contributes a small portion of the entire requests. we rank terms and documents according to their request frequencies and plot the distributions in Figure 2 where Yaxis shows the log-scaled request frequency, while $\mathrm{X}$-axis shows the log-scaled ranking of the request, sorted by their frequencies. The distributions shown in Figure 2 are close to straight lines, suggesting the existence of the Zipf distribution. This finding shows that system cache can improve the service efficiency, considering some query terms and documents are highly popular. If results are stored in a high-speed storage device or in main-memory, a large portion of requests can be answered without accessing the indices or the data store.

The above study does not tokenize query terms. However, many queries have some popular terms in common. Remember for most search engines the query service will tokenize the query string into separate terms and send them to the indices. In addition, full-text indices are arranged as inverted lists corresponding to individual terms. Therefore, inverted lists of popular single-terms can be cached as long as we find similar Zipf distribution for single term frequencies. We examine the logs and plot the finding in Figure 2(c), from which we can see the existence of an approximate straight line, confirming our expectations. 


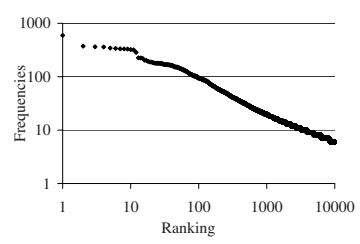

(a) Query term distribution

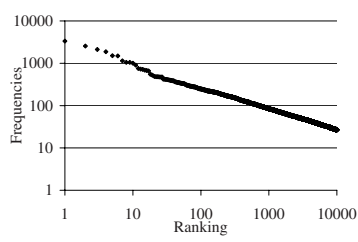

(b) Retrieved document distribution

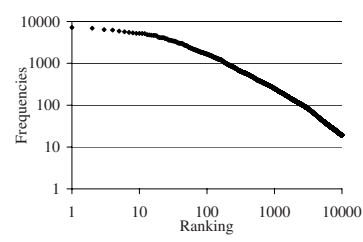

(c) Tokenized query term distribution

Fig. 2. Log-log scale request distribution

\subsection{Request Locality}

Previous studies indicate that there is a high shareness in user requests [10[13]16]. However, the effectiveness of a system cache will be greatly decreased without temporal locality in highly repetitive requests. To answer this question, we analyze the logs to find request localities.

Firstly, we try to reveal the distance between subsequent resubmission of same requests, where distance represents the number of other requests in the interval of a request resubmission. Figure 3(a) and 3(b) shows the distance distribution (in log-log scale) for document query and retrieval respectively. From the plots we can see that most repeated requests have a relatively small distance. In other words, the same requests are often issued with small intervals. This statistics suggest that if we cache temporary query results or documents, it has a high possibility that the cached data will be requested in a short period of time. With a good cache replacement policy deployed, high cache hit rate can be expected.
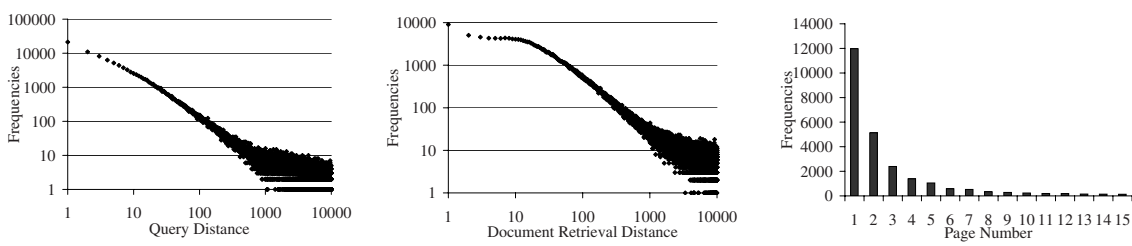

(a) Log-log scale query term (b) distance

(b) Log-log scale document (c)

(c) Browsed result page numretrieval distance ber

Fig. 3. Request locality distributions

We also study user behaviors in browsing paged query results. Previous web studies [3] reveal that users are reluctant to browse pages other than the first one. We conduct the analysis to find the probability for a user to view following pages. The distribution of requested page number is plotted in Figure 3(c), from which we can see most users are only interested in the top 3 pages, which contributes to $75.33 \%$ browse requests. Hence, if we consider prefetching result pages, the scope should not exceed the first 3 ones. 


\subsection{Correlation Studies}

If the system can capture a user's interest on-the-fly from previous operations and retrieve relevant documents beforehand, the user can acquire the documents instantaneously as he clicks the document link. Correlation studies can help us in predicting the possible relevant documents in the repository. For example, if we find users have a high probability to view or download $d_{i}$ after querying $Q, d_{i}$ probably is a good candidate document for the user. We study the correlation between issued queries and the documents that are browsed afterwards. We give the correlation analysis of all 334, 731 distinct logged query strings in Table 2

Table 2. Correlation for query-document $(Q-D)$ pairs

\begin{tabular}{|l|l|l|}
\hline Correlation & $Q-D$ Pairs & Unpopular $Q$ Percentage \\
\hline $1 \%$ & 221,981 & $79.13 \%$ \\
\hline $5 \%$ & 41,718 & $86.61 \%$ \\
\hline $10 \%$ & 18,903 & $90.72 \%$ \\
\hline $50 \%$ & 2,381 & $95.38 \%$ \\
\hline
\end{tabular}

As we look into the queries that have high correlation with documents, we find most queries (90.72\% for $10 \%$ correlational probability or higher, for instance) are unpopular ones, whose cumulative query frequency does not exceed 5 in the logged time interval. This result suggests that if a correlation-based prefetch is supported, it can work as a good supplementary method for ordinary cache mechanisms, which basically serve popular queries.

Correspondingly, we also analyze the correlation between document retrieval requests, which would reveal the relationship between documents. The same procedure is applied to all 393,416 distinct document retrieval requests. The results are given in Table 3

Table 3. Correlation for document-document $\left(D_{1}-D_{2}\right)$ pairs

\begin{tabular}{|l|l|l|}
\hline Correlation & $D_{1}-D_{2}$ Pairs & Unpopular $D_{1}$ Percentage \\
\hline $1 \%$ & 85,695 & $81.68 \%$ \\
\hline $5 \%$ & 6,875 & $84.41 \%$ \\
\hline $10 \%$ & 2,634 & $86.82 \%$ \\
\hline $50 \%$ & 298 & $93.10 \%$ \\
\hline
\end{tabular}

Deeper analysis into the contents of involved papers shows that many highly correlated papers have inherent relationships, including the same author papers, cited-citing pairs, and semantically similar papers. For the limitation of pages, detailed analysis is not included. 


\subsection{Summary}

In this section, an analysis has been performed on the logged user requests. In summary, we find the existence of Zipf distributions in user query terms and retrieved documents. A small portion of hot requests contributes a large portion of the entire traffic. The follow-up locality study shows that for these hot requests, usually they have a high temporal locality. Hence, the results demand a well-tuned cache for CiteSeer to improve the system's performance. Single-term study also suggests caching popular inverted lists. Supplementarily, our correlation study suggests that correlation-based prefetch can determine in advance what documents to be retrieved and reduce the response time of unpopular requests.

\section{Cache Framework}

Based on the conclusion in Section 2, we derive our design for the cache framework of CiteSeer ${ }^{\mathcal{X}}$. In this section, we first discuss what unit should be cached in Section 3.1 For each unit type, the cache is segmented and appropriate replacement policies are employed (Section 3.2). Hybrid cache framework architecture is given in Section 3.3 Finally, we discuss the implementation issues for CiteSeer ${ }^{\mathcal{X}}$.

\subsection{Caching Unit}

The first question that needs to be answered in developing a cache scheme is: what need to be cached? Look at the generic service process for CiteSeer to fulfill a user request. Basically, multiple modules and data sources in the system are involved. Based on request types, we can cache contents in multiple levels. First, our single-term analysis indicates it would be great if we can store indices in high-speed storage devices or main-memory. However, due to the cost concerns and the huge size of indices, it is unrealistic to store all indices in the cache. However, it is easy to cut out a small portion of the index (inverted lists) so that it can fit the cache size. For example, the well-known Lucene 5 library supports such operations and provides in-memory index facility. We call this type of cache as Index Cache.

Another possibility to improve document query performance is to cache result list pages, which we call Result Cache. The unit for this sort of cache is the alreadygenerated result page. Although the result cache serves the same goal as the index cache, it has its own pros and cons. The advantage is that the result cache can save more storage spaces. Also, as we find a result cache hit, the page can be returned instantaneously without looking up the index. However, the result cache can only answer precisely identical queries. One entry in the index cache can be shared by multiple queries.

The third level of cache (Document Cache) is to help improve document retrieval efficiency. Here, the document detail page is wrapped with other auxiliary data (PDF paper, bibtex file, etc) and stored in the cache to avoid invoking related algorithms and unnecessary I/O requests.

\footnotetext{
${ }^{5}$ http://lucene.apache.org
} 


\subsection{Cache Replacement Policy}

Previous search engine cache designs generally are tailored to improve service efficiency for popular requests, with only one cache management policy for all records. In Section 2.3. we observed the Zipf-like distributions of queried terms and retrieved documents. Roughly speaking, the Zipf-like distribution has a head for most popular (hot) requests, with a long tail (unpopular requests). The body of the distribution includes some moderately-popular requests. Observing this, we can cut the distribution into three sections with two frequency threshold parameters $t_{1}$ and $t_{2}$, where $t_{1}$ separates the head section, while $t_{2}$ separates the tail. The value of the parameters are dependent upon the distribution and the cache setting, which will be discussed in Section 3.4

Because request frequencies are dramatically different in each distribution segment, it is helpful to treat them differently, employing separate cache management policies for them. This approach can improve the flexibility in managing the cache so that the system performance can be tuned. For the hot requests, we use a static cache policy, with the cached items always resident in the cache. For the moderately-popular requests, we implement a dynamic cache replacement policy, in which some well-known cache replace algorithm (LRU, SLRU, etc [5]) is used. For unpopular requests, we do not reserve cache space for them. However, a prefetch buffer is included in the cache to store prefetched data based on correlation statistics. As we have seen earlier, the prefetch is very effective for unpopular requests. The one-policy approach can be viewed as an extreme case of the hybrid cache design.

For the three components of caches, three previously observed Zipf-like distributions are used. To be specific, the tokenized query term distribution is used for the index cache, the document query term distribution for the result cache, and the document retrieval identifier distribution for the document cache. Each distribution has its own frequency threshold parameters, which are represented as $t_{i_{1}}$ and $t_{i_{2}}$, where $i \in\{$ index, result, document $\}$.

Based on the above discussions, we have the conceptual view of the hybrid caching framework for CiteSeer ${ }^{\mathcal{X}}$, as shown in Figure 4

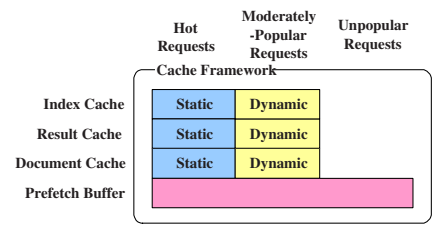

Fig. 4. Conceptual view of the hybrid cache framework

\subsection{Architectural Design}

As an important part of the query engine in $\mathrm{CiteSeer}^{\mathcal{X}}$, the cache framework captures incoming requests before they access the data repository. The whole process is briefly illustrated in Figure 5. When receiving a request, the request classifier determines the request type based on the encoded URL. Afterwards, the cache lookup module checks 


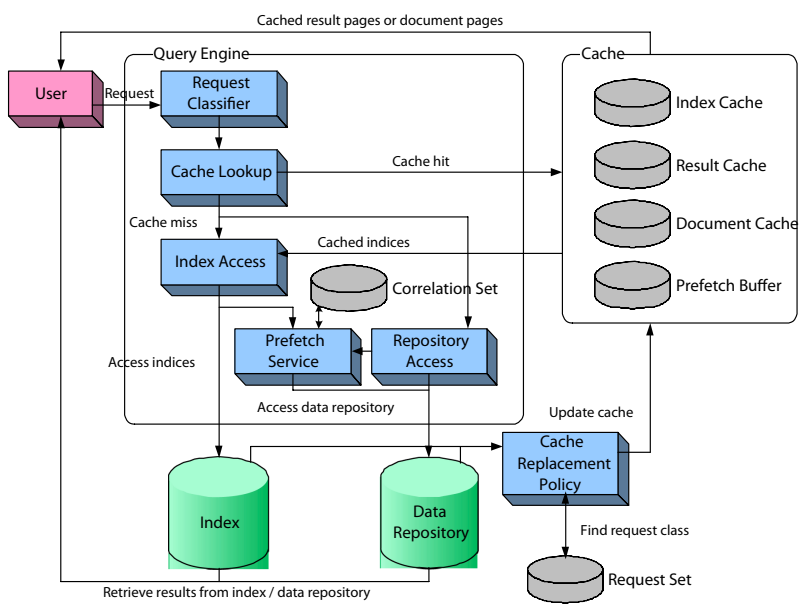

Fig. 5. The workflow in answering a user request

the cache for the results of the request. If a cache hit is found, either the result page can be returned to the user instantly or the cache contains some index entries for the query request. If it happens to be the latter case, partial resolved results are sent to the index access module for further processing. If there is a cache miss, the query engine directs the request to the index access module or the data repository access module. Prefetch can be triggered by previous requests, which access a built-in correlation set to find relevant documents, and store them in the prefetch buffer. The prefetched documents are removed from the buffer as long as the current user session terminates or another query is issued by the same user. Also, our analysis confirms that after issuing a query to CiteSeer, a user has a high possibility to access the second and third result list page. The two pages, if available, are prefetched when the user is browsing the first result list page until the session ends or the arrival of a new query. After fulfilling a user's request by index or data repository, the cache replacement policy works to check if the cache needs to be updated. A request set, resident in a high-speed local device, classifies the request into a popularity class (popular, moderately-popular, unpopular) and thus directs the request to an appropriate cache. The victim cached data entry is removed.

\subsection{Implementation Issues}

Suppose the available cache size is $S$ in total, an important decision to be made during implementing the hybrid cache is to determine how to divide $S$ for each cache component. Actually, the number of entries in the prefetch buffer is the same as the number of active users in the system, because each user has a collection of prefetched documents based on previous requests. The active user number is normally no more than 50 in CiteSeer. Hence, its size is negligible to the entire cache. We use a set of parameters, $f_{x}(x \in\{$ index, result, document $\})$, to denote the size percentage of $S$ reserved for each cache component. The value of $f_{x}$ is within the range $[0,1]$ and the sum of all $f_{x}$ equals to 1 . 
The optimized values of $f_{x}$ are influenced by request distributions. Remember in Section 3.2 two thresholds are used to separate each request distribution into three parts. Actually, parameter $t_{1}$ decides the number of popular requests for each frequency distribution. As a result, the static cache size in each component is heavily depended on $t_{1}$. In the experimental settings, we only restrict the size reserved for each cache component. Parameter $t_{1}$ determines how many units should be cached in each static cache, and thus decides the actual static cache size. The remainder storage is used for the dynamic portion.

CiteSeer is an autonomous system that keeps obtaining new documents from Internet. System workload also varies over time. As the statistical analysis grows out-of-date, the effectiveness of the cache will be decreased. It is necessary to update the statistics in the system over a period of time. The update process mainly includes summarizing user request, updating request set, re-calculating request correlation, updating correlation set, and tuning cache sizes. These tasks can be performed offline in another server. Updated data can be switched to the public server during system maintenance period.

\section{Performance Evaluation}

\subsection{System Setup}

We develop a test query engine, which includes the proposed cache framework, for the evaluation. Actual CiteSeer data collection and real logged usage traces are used in the experimental platform. CiteSeer's internal algorithms are also included to dynamically generate output results. Lucene is used to index documents. This testing platform needs to communicate with CiteSeer for data and services, which increases the response time. This extra overhead remains almost constant regardless the workload and query processing strategy. The dataset is stored in a SUN StorEdge 3511 FC array with SATA hard drives. We implement the cache on a workstation (CPU: 1 AMD Opteron Processor 252, 4GB Main Memory, OS: Red Hat). Index cache is an in-memory cache. Result cache and document cache are stored in local high-speed disks. We use the popular

Table 4. Default experiment implementation parameters

\begin{tabular}{|c|c|}
\hline System parameter & Default value \\
\hline Cache size $(S)$ & $2 G B$ \\
\hline Prefetch buffer size & $50 M B$ \\
\hline Correlation threshold & 0.1 \\
\hline Cache component & Size portion \\
\hline Index cache & $50 \%$ \\
\hline Result cache & $12.5 \%$ \\
\hline Document cache & $37.5 \%$ \\
\hline Distribution & Frequency threshold \\
\hline Tokenized term & $t_{\text {index }_{1}}=1000, t_{\text {index }_{2}}=10000$ \\
\hline Query term & $t_{\text {result }_{1}}=300, t_{\text {result }_{2}}=3000$ \\
\hline Retrieved document & $t_{\text {document }_{1}}=1000, t_{\text {document }_{2}}=10000$ \\
\hline
\end{tabular}


cache replacement algorithm, $L R U$, as an example to manage the dynamic caches. The default parameter values we set for our experiments are given in Table 4

Two workloads are applied in our experiments. The first workload (actual trace) uses another piece of system usage $\log (38,590$ queries and 212, 269 document retrievals) to mock user clients. The other workload (uniform trace) is randomly generated from the request corpus, without considering the popularity. The averaged response time and cache hit rate are used for performance comparison.

\subsection{Effects of Caching}

As the first experiment, we study CiteSeer's performance in answering requests under different system settings. The actual trace and uniform trace are both used for comparison, whose results are given in Figure 6 .

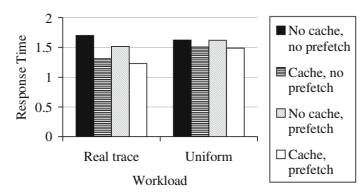

(a) Response time

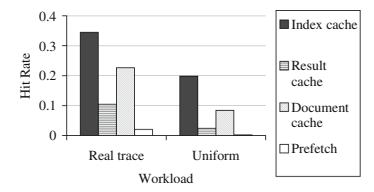

(b) Cache and prefetch hit rate

Fig. 6. Cache effect study

Figure 6(a) compares the system's response time under various running conditions. It is obvious that using cache can improve the performance dramatically $(27.65 \%$ improvement). Considering the fact that document retrieval requests contribute a dominating percentage in the actual trace, prefetch technique can further improve the response time. Also, it is shown that our caching mechanism works better for the actual workload than the uniform workload. This is because there exists a strong Zipf-like distribution in request semantics in the actual trace, which is absent in the uniform workload. In the following experiments, only actual trace is used.

Figure 6(b) gives the cache hit rates for each cache component. Index cache has a higher hit rate than any other cache component, because some queried terms are widely shared by many queries. In addition, document cache is more effective than the result cache, suggesting temporal locality in requested documents is stronger than queries, which can be observed from Figure 2 as well.

\subsection{Cache Size Effects}

In this section, we change the cache size parameter $S$. The size of the prefetch buffer and the fraction of each cache component is fixed.

It is expected that a larger cache size can bring better cache hit rate, and thus returns a shorter response time. This expectation is confirmed from the above figures. However, huge cache size is inefficient in cost and storage. As we can see from Figure 7(a) the response time does not change too much as the cache size reaches $1 G B$, showing a 


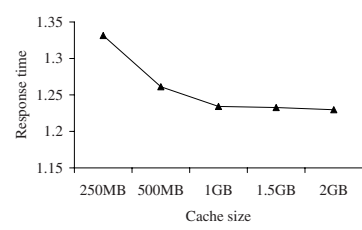

(a) Response time

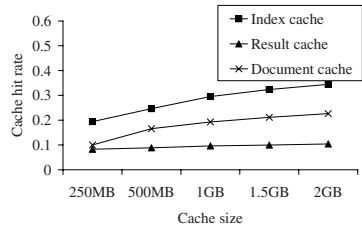

(b) Cache hit rate

Fig. 7. Cache size effect study

reasonably good cache performance can be achieved without a larger cache. Figure 7 (b) indicates when the cache size reaches $1 G B$ or larger, all cache hit rates only increase in a slow speed with the size.

\subsection{Correlation Threshold Effects}

When we construct the correlation set, we use the correlation probability threshold to filter the strong-correlated request pairs. In the default setting, this value is fixed at 0.1 , indicating a $10 \%$ or higher correlation probability is required. In this experiment, we change the threshold value to observe its effect.

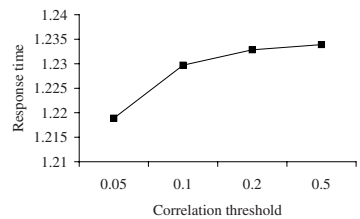

(a) Response time

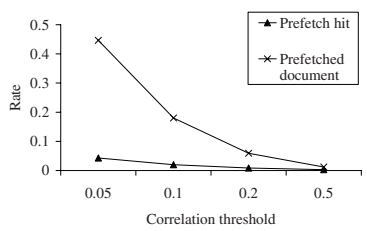

(b) Prefetch hit rate and prefetched document rate

Fig. 8. Correlation threshold effect study

From Figure 8(a), we find a low threshold is very useful in improving the average response time. However, as seen from Figure 8(b) a low threshold means more correlated request pairs are qualified, and thus more documents are prefetched, most of which are not accessed by follow-up requests. When we set the correlation threshold to be 0.05 , a document prefetch rate of 0.45 is reached. This means that on average each request will trigger 0.45 document to be stored in the prefetch buffer. However, the hit rate at the time is only around 0.05 , meaning most of them will be replaced from the buffer without any access. The above analysis indicates us to control the correlation threshold at a reasonable level to optimize the performance.

\subsection{Size Parameter Tuning}

In this section, we investigate the problem of tuning each cache component size, assuming the total cache size is fixed. 


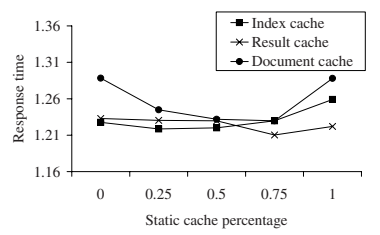

(a) Response time

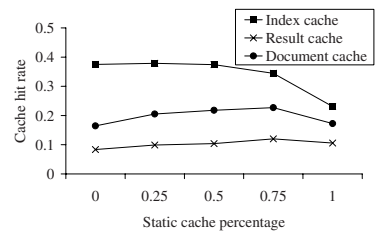

(b) Cache hit rate

Fig. 9. Tuning static cache percentage

In the first experiment, we fix the assigned sizes for each cache component and respectively vary the static cache size in each component. Each curve in Figure 9 gives the study result for a specific cache component. Generally, the three curves are similar in their shapes in Figure 9(a) and Figure 9(b) respectively. It is suggested that the extreme cases (the entire cache uses static or dynamic policy) do not produce the optimal performance. This study also gives evidence in supporting a segmented cache management policy for search engines. It is also found that the optimal points on the curves are different for each cache component, indicating the necessity to study the distributional request feature behind each type of cache to optimize it.

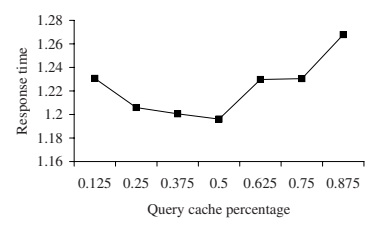

(a) Response time

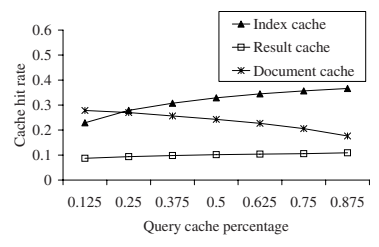

(b) Cache hit rate

Fig. 10. Tuning query cache percentage

In the next study, we keep the static-dynamic ratio in each cache component constant and try to tune the storage reserved for the query caches (result cache and index cache) and the document cache. We perform this analysis because the two targets serve different request types. From Figure 10(b) we can clearly see how the two cache categories compete for cache space. The document cache performance is sacrificed if we increase the query cache sizes, and vice versa. Overall, the system reaches its best performance in the middle of the curve shown in Figure 10(a) meaning the cache should be divided approximately evenly for the query caches and the document cache. This value is affected by the composition of the request stream, in which we find the document retrieval requests are approximately 5 times as many as the query requests in CiteSeer.

Finally, we study the details in the query caches by modifying sizes reserved for the index cache and the result cache. The results (Figure 11) indicate the optimal size ratio is not reached in the extreme case as well. We can see that the hit rates for both caches do not change dramatically as long as their sizes are not extremely small. Thereafter, the response time is not very sensitive to the size ratio between the two cache components. 


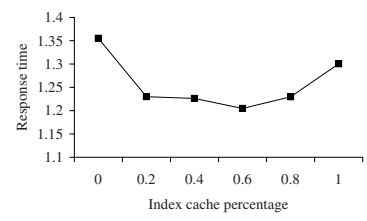

(a) Response time

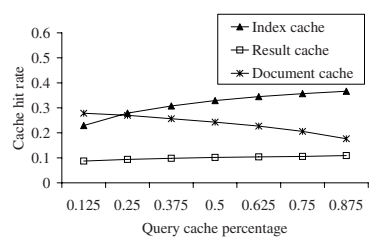

(b) Cache hit rate

Fig. 11. Tuning index cache percentage

\section{Related Work}

A large number of works have been performed to study user behaviors in search engines by analyzing logged data. As one of the first few papers discussing search engine user behavior, [3] analyzed query logs of Fireball, a popular German search engine, showing a large portion of users only browse the first page of returned results. [17] studied Altavista search engine and found the majority of the users submitted only one query before the session ended. [13] shows the existence of locality in the queries for Excite search engine. The results are later confirmed by another study [16]. A late work on Altavista [10] shows over $67 \%$ of the logged queries are requested only once, while other few queries are requested frequently. Again, the Zipf distribution is observed.

Based on these observations, server-side cache management mechanism is adopted to improve system performance. Basically, two types of caches are proposed [16]. One choice is the Result Caching [9]13]. The previously processed query results are cached to answer future identical queries. The alternative approach is to cache inverted index other than results. The idea of inverted index caching is to extract a portion of the entire index to store in the cache [4]. Corresponding cache replacement policy that is based on access semantic is enforced. Meanwhile, the application is able to support dynamic query evaluation based on cache contents. [16] exploits the opportunity to combine the previously addressed two caching types in the search engine TodoBR. [12] found it is often observed that large web search engines are related to multiple data sources. Hence, a new intermediate level is added to cache intersections or projections of the frequently occurred term pairs, which contributes to the three-layer architecture. Furthermore, [18] suggests a hybrid composition of search engine cache which maintains a static set of statically locked cache entries and a dynamic set which contains replaceable cache entries. In addition, a speculative result page prefetching strategy is added to improve the hit ratio. Prefetching technique is also referred in other web applications [14 15].

Another popular cache study direction is in the infrastructure level. In summary, based on the usage patterns, it is interesting to find the best place to cache web contents [1920]. Cache is widely used in other research domains. [811] studied how to maintain caches in mobile environment, with limited resources. [1] uses profiles to manage caches, which can be used for personalized services. 


\section{Conclusion}

In this paper, we present our initial design of a cache framework for a scientific literature search engine, CiteSeer. Our workload analysis on the usage logs indicates characteristics of requests in such a system, including temporal localities and the correlation of requests. Based on our findings, the proposed system cache is comprised of multiple components, which include index cache, result cache and document cache. Correlationbased prefetch is provided to further improve the document retrieval efficiency. Experimental results show that our caching mechanism produces dramatic improvement in the system's response time.

Our future work will investigate the research interests of users to decrease the request ambiguity and improve the service precision. Another future research direction is to develop an adaptive cache management policy to tune cache parameters in real time.

\section{Acknowledgements}

We gratefully acknowledge partial support from Microsoft Research, NSF and NASA for CiteSeer project. Wang-Chien Lee was supported by NSF under Grant No. IIS0328881, IIS-0534343 and CNS-0626709.

\section{References}

1. Cherniack, M., Galvez, E.F., Franklin, M.J., Zdonik, S.B.: Profile-driven cache management. In: ICDE, pp. 645-656 (2003)

2. Giles, C.L., Councill, I.G.: Who gets acknowledged: measuring scientific contributions through automatic acknowledgement indexing. In: Proceedings of the National Academy of Sciences. vol. 101(51), pp. 17599-17604 (2004)

3. Hölscher, C.: How internet experts search for information on the web. In:WebNet (1998)

4. Jónsson, B.T., Franklin, M.J., Srivastava, D.: Interaction of query evaluation and buffer management for information retrieval. In: SIGMOD Conference, pp. 118-129 (1998)

5. Karedla, R., Spencer Love, J., Wherry, B.G.: Caching strategies to improve disk system performance. IEEE Computer 27(3), 38-46 (1994)

6. Lawrence, S., Bollacker, K.D., Giles, C.L.: Indexing and retrieval of scientific literature. In: CIKM, pp. 139-146 (1999)

7. Lawrence, S., Giles, C.L., Bollacker, K.: Digital libraries and Autonomous Citation Indexing. IEEE Computer 32(6), 67-71 (1999)

8. Ken C., Lee, K., Lee, W.-C., Zheng, B., Xu, J.: Caching complementary space for locationbased services. In: EDBT, pp. 1020-1038 (2006)

9. Lempel, R., Moran, S.: Optimizing result prefetching in web search engines with segmented indices. In: VLDB, pp. 370-381 (2002)

10. Lempel, R., Moran, S.: Predictive caching and prefetching of query results in search engines. In: WWW, pp. 19-28 (2003)

11. Lim, S., Lee, W.-C., Cao, G., Das, C.R.: A novel caching scheme for improving internetbased mobile ad hoc networks performance. Ad Hoc Networks 4(2), 225-239 (2006)

12. Long, X., Suel, T.: Three-level caching for efficient query processing in large web search engines. In: WWW, pp. 257-266 (2005) 
13. Markatos, E.P.: On caching search engine query results. Computer Communications 24(2), 137-143 (2001)

14. Min-You, Y.J.: Web prefetching: Costs, benefits and performance.

15. Nanopoulos, A., Katsaros, D., Manolopoulos, Y.: Effective prediction of web-user accesses: a data mining approach. In: Kohavi, R., Masand, B., Spiliopoulou, M., Srivastava, J. (eds.) WEBKDD 2001 - Mining Web Log Data Across All Customers Touch Points. LNCS (LNAI), vol. 2356, Springer, Heidelberg (2002)

16. Saraiva, P.C., de Moura, E.S., Fonseca, R.C., Wagner Meira Jr., Ribeiro-Neto, B.A., Ziviani, N.: Rank-preserving two-level caching for scalable search engines. In: SIGIR, pp. 51-58 (2001)

17. Silverstein, C., Henzinger, M.R., Marais, H., Moricz, M.: Analysis of a very large web search engine query log. SIGIR Forum 33(1), 6-12 (1999)

18. Silvestri. F.: High performance issues in web search engines: Algorithms and techniques. Ph.D. dissertation. UniversitPá degli Studi di Pisa—Facoltà di Informatica, Pisa, Italy.

19. Wong, T.M., Wilkes, J.: My cache or yours? making storage more exclusive. In: USENIX Annual Technical Conference, General Track, pp. 161-175 (2002)

20. Xie, Y., O'Hallaron, D.R.: Locality in search engine queries and its implications for caching. InINFOCOM (2002) 